\title{
BMJ Open Association of antenatal corticosteroids with morbidity and mortality among preterm multiple gestations: meta- analysis of observational studies
}

\author{
Dongxin Lin, ${ }^{1,2}$ Dazhi Fan, ${ }^{1,2}$ Gengdong Chen, ${ }^{1,2}$ Caihong Luo (D) , ${ }^{2}$ Xiaoling Guo, ${ }^{1,2}$ \\ Zhengping Liu (iD) ${ }^{1,2}$
}

To cite: Lin D, Fan D, Chen G, et al. Association of antenatal corticosteroids with morbidity and mortality among preterm multiple gestations: metaanalysis of observational studies. BMJ Open 2021;11:e047651. doi:10.1136/ bmjopen-2020-047651

- Prepublication history and additional material for this paper are available online. To view these files, please visit the journal online (http://dx.doi org/10.1136/bmjopen-2020047651).

Received 07 December 2020 Accepted 07 September 2021

Check for updates

(c) Author(s) (or their employer(s)) 2021. Re-use permitted under CC BY-NC. No commercial re-use. See rights and permissions. Published by BMJ.

${ }^{1}$ Foshan Institute of Fetal Medicine, Southern Medical University Affiliated Maternal \& Child Health Hospital of Foshan, Foshan, Guangdong, China ${ }^{2}$ Department of Obsterics, Southern Medical University Affiliated Maternal \& Child Health Hospital of Foshan, Foshan, Guangdong, China

Correspondence to

Dr Caihong Luo;

lawcaihong@outlook.com

\section{ABSTRACT}

Objective This meta-analysis aimed to assess the efficacy of antenatal corticosteroids (ACS) on morbidity and mortality among preterm multiple pregnancies.

Methods The PubMed, Embase, Web of Science and Cochrane Library databases were searched for studies investigating the outcomes among preterm multiple gestations following to ACS, from their inception to 1 November 2020. Two authors independently performed the study selection, risk of bias assessment and data extraction. The primary outcomes were respiratory distress syndrome (RDS) and mortality and secondary outcomes included intraventricular haemorrhage (IVH), periventricular leukomalacia (PVL), necrotising enterocolitis, retinopathy of prematurity and bronchopulmonary dysplasia. Pooled ORs were obtained using random effects models. Subgroup analyses were performed to explain heterogeneity by ACS completeness, administration-to-delivery intervals ( $\leq 7$ days) and single or multicentre.

Results A total of 16 observational studies with 36973 newborns were included in the meta-analysis. ACS treatment was associated with a reduction in RDS (OR $0.66 ; 95 \% \mathrm{Cl} 0.54$ to $\left.0.82 ; I^{2}=91.4 \% ; p<0.001\right)$, mortality (OR $0.64 ; 95 \% \mathrm{Cl} 0.50$ to $0.81 ; I^{2}=85.9 \% ; p<0.001$ ), IVH (OR 0.67; $95 \% \mathrm{Cl} 0.54$ to $\left.0.83 ; \mathrm{I}^{2}=77.4 \% ; \mathrm{p}<0.001\right)$ and PVL (OR $0.65 ; 95 \% \mathrm{Cl} 0.47$ to $0.92 ; l^{2}=75.5 \%$; $p<0.001)$. Subgroup analyses showed ACS completeness, administration-to-delivery interval and multicentre study affected these associations.

Discussion ACS may be beneficial for reducing the risks of RDS, mortality, IVH and PVL among preterm multiple gestations. The efficacy of ACS could be affected by ACS completeness and administration-to-delivery. More robust evidence on the efficacy of ACS treatment among multiple gestations is warranted.

\section{INTRODUCTION}

Preterm birth (PTB) is one of the most common complications and the leading cause of perinatal morbidity and mortality among multiple gestations. ${ }^{1-3}$ In the USA, the rates of PTB (<37 weeks, 60.32\%) and early PTB (<34 weeks, $19.52 \%$ ) among twin pregnancies were substantially higher than those among
Strengths and limitations of this study

- This is a meta-analysis investigating the benefits of antenatal corticosteroids (ACS) in preterm multiple gestations since this group is not addressed separately in previous systematic reviews.

- We aimed to generate pooled estimates of effect of ACS on important outcomes of newborns in multiple pregnancies.

- No evidence of randomised controlled trials was available; thus, we focused on observational studies and synthesised the evidence in real world.

- The high heterogeneity remained in some subgroups, which suggested the presence of unexplained heterogeneity.

singletons $(10.02 \%$ for PTB $<37$ weeks and $2.75 \%$ for $\mathrm{PTB}<34$ weeks) in $2018 .{ }^{4}$ The rate of PTB among multiple gestations remains at a high level in many countries. ${ }^{5-8}$

Improving the prognosis of premature newborns has become an important issue in clinical practice. Liggins and Howie first demonstrated the advantage of treatment with antenatal corticosteroids (ACS) to promote lung maturation and reduce respiratory distress syndrome (RDS) among premature infants. ${ }^{9}$ The effect of a single course of ACS prior to PTB on reduction not only in RDS but also in other complications related to prematurity have been ascertained by the latest Cochrane review. ${ }^{10}$ Although this prophylactic treatment is recommended for potential premature births by the American College of Obstetricians and Gynecologists ${ }^{11}$ and the Royal College of Obstetricians and Gynecologists, ${ }^{12}$ there remains a controversy regarding multiple gestations. ${ }^{13}{ }^{14}$ While the efficacy of ACS treatment in singletons is supported by abundant evidence, the current evidence in multiple gestations is limited and less consistent. ${ }^{15-25}$ 
Therefore, we aimed to perform a meta-analysis of observational studies investigating the efficacy of ACS treatment on neonatal mortality and morbidity among multiple PTB.

\section{METHODS}

The meta-analysis was based on the Preferred Reporting Items for Systematic Reviews and Meta-analyses (available in online supplemental table S1) ${ }^{26}$ and Meta-analysis of Observational Studies in Epidemiology Guidelines. ${ }^{27}$

\section{Patients and public involvement statement}

No individual patient was involved in the study design, conduct, reporting or dissemination.

\section{Literature search and study selection}

We conducted a systematic literature search in the PubMed, Embase, Web of Science and Cochrane Library databases using a search strategy that combined the following keywords/MeSH terms: (1) steroids, corticosteroids, betamethasone, dexamethasone, hydrocortisone, (2) preterm, premature, (3) prenatal, antenatal, antepartum and (4) twins, multiple gestation, twin pregnancy. Articles in English were searched from their inception to 1 November 2020. Search strategies for each database are shown in online supplemental table S2. All records retrieved from literature search were imported into Endnote and duplicate records were first removed. Then, the titles and abstracts of the remaining studies were screened for potential eligibility based on the search strategies. Third, full texts were reviewed based on the inclusion criteria. In addition, the references were manually searched in the procedure of full-text review.

\section{Eligible criteria}

Studies that met the following inclusion criteria were eligible for the current meta-analysis: (1) the study evaluated the association of ACS treatment with morbidity and/or mortality (comparison between ACS and non-ACS users); (2) the outcomes assessed in individual studies were within the scope of outcomes of interest in the current meta-analysis and (3) the study provided results in subgroups of multiples if it enrolled both singleton and multiple pregnancies. Studies were excluded if they did not provide relevant data on the incidence or ORs of outcomes of interest between ACS and non-ACS users. Articles published as conference abstracts, letters to the editor or systematic reviews were also excluded due to insufficient information. We imposed no restriction on the study design in current meta-analysis in the procedure of study selection.

\section{Risk of bias and evidence quality assessment}

The risk of bias of the included studies was assessed based on the Newcastle-Ottawa Scale (NOS) (http://www.ohri. $\mathrm{ca} /$ programs/clinical_epidemiology/oxford.asp), which consists of eight items for three subscales (four items for selection, one item for comparability and three items for outcome). The maximum score is 9 (2 for the comparability item and 1 for each of the other items). A score of 5 or below indicated a high risk of bias, 6-7 points indicated an intermediate risk of bias and 8-9 points indicated a low risk of bias. We applied the Grading of Recommendations, Assessment, Development and Evaluation (GRADE) system to assess the overall quality of evidence for each outcome across studies. ${ }^{28}$ According to the guidelines, observational studies started as low-quality evidence. The evidence quality could be downgraded based on study limitations, imprecision, inconsistency, indirectness and publication bias, whereas it could be upgraded based on a larger magnitude of effect, a doseresponse gradient and all plausible confounding which would reduce a demonstrated effect.

\section{Data extraction}

The following information regarding study characteristics and primary results was extracted by using a predesigned form: the first author, year of publication, country, study design, sample size, population characteristics, exclusion criteria, gestational age at delivery, details of ACS administration (type of drug, dose, ACS completeness and administration-to-delivery interval), outcomes reported, incidence or adjusted ORs of each outcome. The results of the subgroup analysis based on complete ACS and administration-to-delivery were also extracted if available. Complete ACS was defined as four $6 \mathrm{mg}$ doses of intramuscular dexamethasone at 12-hour intervals or two $12 \mathrm{mg}$ doses of intramuscular betamethasone at a 24-hour intervals. Studies were categorised as 'complete ACS' when they only included cases with complete ACS or reported results in 'complete ACS' subgroups. Studies that only included cases with administration-to-delivery intervals $\leq 7$ days or provided results in such subgroups were categorised as 'administration-to-delivery intervals $\leq 7$ days'. Those without information on ACS completeness or the timing of therapy were categorised as 'unclear'. Two authors (GC and FD) independently performed study selection, risk of bias assessment and data extraction and any disagreements were resolved by the discussion.

\section{Outcomes of interest}

The primary outcomes in the current meta-analysis were RDS and mortality while secondary outcomes included intraventricular haemorrhage (IVH), periventricular leukomalacia (PVL), necrotising enterocolitis (NEC), retinopathy of prematurity (ROP) and bronchopulmonary dysplasia (BPD). These outcomes were defined by the authors of individual studies and were evaluated before hospital discharge. Overall, RDS was diagnosed based on clinical manifestations (cyanosis, grunting, retractions and tachypnoea), blood gas and radiographic chest findings, need for oxygen or lung surfactant administration; IVH (graded by Papile et al criteria $^{29}$ ) and PVL were diagnosed based on cranial ultrasonography, MRI or autopsy; NEC (graded by Bell's criteria ${ }^{30}$ ) was diagnosed based on the clinical and radiological manifestations; ROP 
(graded by the International Classification of $\mathrm{ROP}^{31}$ ) was diagnosed based on ophthalmological examination; and BPD was diagnosed based on duration of supplementary oxygen, radiographic or histological findings.

\section{Statistical analysis}

The meta-analysis was performed by using Stata V.15.1 software (StataCrop). A fixed effects model was used to calculate a pooled OR if a study only provided results on subgroups and this pooled OR was used for metaanalysis. ${ }^{32}$ A random effects models with the MantelHaenszel method was used to calculate the pooled ORs and $95 \%$ CIs since we cannot discount a small study by giving it a very small weight (the way in a fixed effect model) and want to be sure that all the effect sizes are represented in the summary estimate. ${ }^{33}$ The heterogeneity across the included studies was assessed by Cochran $\mathrm{Q}$ test and $\mathrm{I}^{2}$ statistics. The heterogeneity with a $\mathrm{p}<0.1$ for Cochran Q test or a $\mathrm{I}^{2}$ statistics $>50 \%$ was considered statistically significant. To explore the potential sources of heterogeneity, we performed subgroup analyses based on ACS completeness, administration-to-delivery interval and study design (multicentre vs single centre). Publication bias was evaluated by using Begg's and Egger's tests.
A $\mathrm{p}<0.05$ indicated potential bias. A trim-and-fill procedure was also performed to further assess the possibility of publication bias when a significant $p$ value was found for Begg's and/or Egger's tests. To evaluate the stability of the pooled results, sensitivity analyses were carried out by omitting individual studies at a time.

\section{RESULTS}

\section{Study selection}

The process of study selection is outlined in figure 1 . A total of 590 records were obtained in the literature search. Duplicate $(n=161)$ and irrelevant records $(n=373)$ were removed after title and abstract screening, leaving 56 studies for full-text review. Additionally, one study was regarded as potentially eligible after manually reference lists. Finally, a total of 16 studies, involving 36973 multiple gestation infants, were included in the metaanalysis. ${ }^{15-25} 34-38$

\section{Characteristics of included studies}

The characteristics are presented in online supplemental table S3. There were 10 retrospective cohort studies, ${ }^{16} 19-2325343738$ population-based studies, ${ }^{17} 1824$

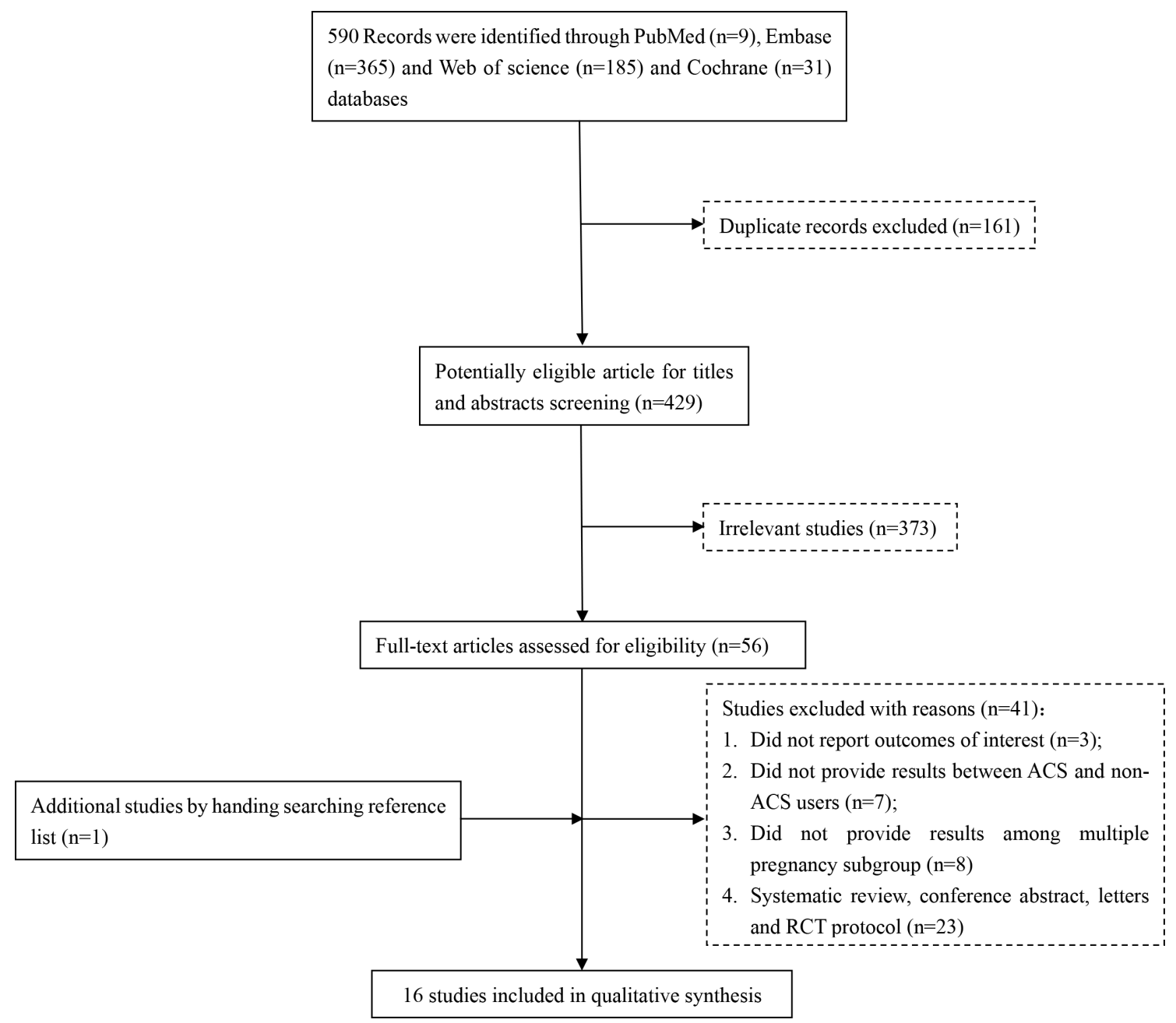

Figure 1 Flow chart of study selection. ACS, antenatal corticosteroids; RCT, randomised controlled trial. 
2 prospective cohort studies ${ }^{35} 36$ and 1 secondary analysis of randomised controlled trial (RCT). ${ }^{15}$ Seven studies had information on the ACS administration-to-delivery interval. ${ }^{16} 192025$ 34-36 Nine studies provided results on the 'complete ACS' subgroup or only enrolled patients with complete ACS treatments. ${ }^{16-21} 2325$ 34-36 The range of gestational age at delivery of the included participants differed across the included studies. Four studies included triplets or higher order pregnancies. ${ }^{21} 222425$ The definition or grading of outcomes (IVH, NEC, ROP and BPD) was not all the same across included studies. Most studies included IVH $\geq$ grade III, NEC $\geq$ grade II and ROP $\geq$ grade III.

\section{Quality assessment of included studies}

Overall, the quality of the included studies was good, with a score ranging from 7 to 9 , based on the NOS (online supplemental table S4). All included studies were derived from non-randomised samples. Seven studies $^{162023343538}$ had a risk of bias on representativeness of the exposed cohort mainly due single-centre sample or small study size. The representativeness of the non-exposed group was considered good in all 16 studies regarding the same origin as the exposed groups. Eight studies ${ }^{15} 171822243738$ had a risk of bias on ascertainment of exposure since these studies did not have specific information on the ACS course. All the studies had no bias with respect to the "demonstration that outcome of interest was not present at start of study' since these outcomes did not occur before birth. All studies except one ${ }^{16}$ show good comparability with controlling important confounders such as gestational age, chorionicity, birth weight and maternal age. All included studies acquired one star in 'assessment of outcome' since the data on outcomes were extracted from medical records or by database linkage. The length of follow-up was acceptable in all studies, as the outcomes were assessed before hospital discharge. Seven studies $^{1720212534-36}$ scored no star in the 'adequacy of follow-up' item due to a lost to follow-up rate $>10 \%$ or due to lacking description.
Meta-analysis of the association between ACS treatment and neonatal outcomes of preterm multiples

Respiratory distress syndrome

Fourteen studies reported results on RDS between ACS and non-ACS groups. The pooled OR was 0.66 (95\% CI 0.54 to $0.82 ; I^{2}=91.4 \% ; p<0.001$ ), indicating that preterm twins with ACS exposure were associated with a lower risk of RDS development (table 1, online supplemental figure S1). In the subgroup analysis, we found that a lower pooled OR of RDS (OR 0.42; 95\% CI 0.26 to 0.68 ) was obtained among participants exposed to complete ACS. A non-significant pooled OR, however, was obtained among those studies that had no information on ACS completeness (OR 0.88; 95\% CI 0.71 to 1.10). In the subgroup analysis by ACS administration-todelivery interval, preterm multiples with ACS treatment with an administration-to-delivery interval of $\leq 7$ days had a lower pooled OR $(0.34 ; 95 \% \mathrm{CI} 0.17$ to 0.70$)$ for RDS. In contrast, those studies including pregnancies with ACS treatment with an administration-to-delivery interval of $>7$ days or having no information on the administration-to-delivery interval obtained a non-significant pooled OR $(0.84 ; 95 \%$ CI 0.69 to 1.02). Single-centre studies obtained a significantly decreased pooled OR $(0.47 ; 95 \%$ CI 0.19 to 0.90$)$, however, multicentre studies did not $(0.82 ; 95 \%$ CI 0.67 to 1.00$)$ (online supplemental table S5). The GRADE assessment indicated that the overall quality of evidence for the risk of RDS was very low due to the very serious inconsistency (online supplemental table S6).

\section{Mortality}

Among 11 studies reporting neonatal mortality, a pooled OR of 0.64 (95\% CI 0.50 to $0.81 ; \mathrm{I}^{2}=85.9 \%$; $\left.<<0.001\right)$ was obtained (table 1, online supplemental figure S2). In the subgroup analysis, the mortality was lower among preterm multiples following to a complete ACS compared with non-ACS multiples (pooled OR 0.41; 95\% CI 0.32 to 0.53 ), but not different when the completeness of treatment was not reported (pooled OR 0.76; 95\% CI 0.56 to 1.01). The mortality was lower among twins with ACS exposure, irrespective of ACS administration-to-delivery intervals. The

Table 1 Meta-analyses of the associations of ACS treatment and neonatal outcomes

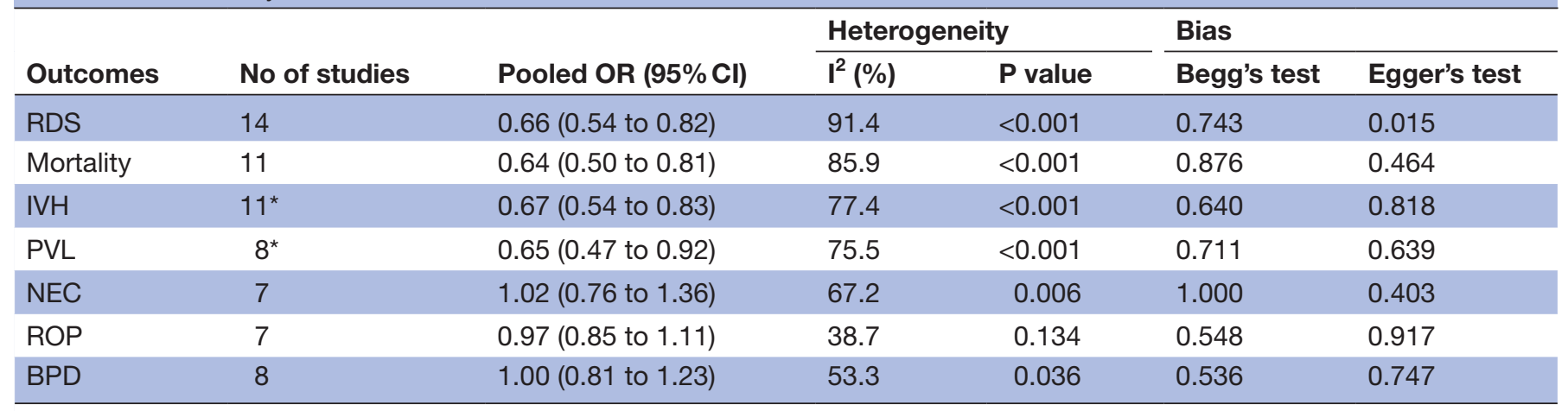

*Two studies assessed IVH and PVL as a composite measure.

ACS, antenatal corticosteroids; BPD, bronchopulmonary dysplasia; IVH, intraventricular haemorrhage; NEC, necrotising enterocolitis; PVL, periventricular leukomalacia; RDS, respiratory distress syndrome; ROP, retinopathy of prematurity. 
pooled OR of mortality was significantly lower among multicentre studies (pooled OR 0.64; 95\% CI 0.50 to 0.82 ) but not among single-centre studies (pooled OR 0.59; 95\% CI 0.24 to 1.43) (online supplemental table S5). The GRADE assessment indicated that the overall quality of evidence for the risk of mortality was very low due to the very serious inconsistency (online supplemental table S6).

\section{IVH and PVL}

There were nine and six studies reporting results on IVH and PVL, respectively. Another two reported results on IVH and PVL as a composite measure. We yielded a decreased pooled OR for IVH (pooled OR 0.67; 95\% CI 0.54 to 0.83 ; $\mathrm{I}^{2}=77.4 \% ; \mathrm{p}<0.001$ ) and PVL (pooled OR $0.65 ; 95 \% \mathrm{CI} 0.47$ to $0.92 ; \mathrm{I}^{2}=75.5 \% ; \mathrm{p}<0.001$ ) (table 1 , online supplemental figure S3-S4). Subgroup analyses revealed that the odds of IVH were lower among multiples exposed to ACS, irrespective of treatment completeness or administration-to-delivery interval. The pooled OR was significant among multicentre studies (pooled OR $0.68 ; 95 \%$ CI 0.55 to 0.85 ) but not among single-centre studies (pooled OR 0.47; 95\% CI 0.09 to 2.41). For PVL, the OR was lower only when mothers received complete ACS (pooled OR 0.41; 95\% CI 0.21 to 0.81 ), delivered within 7 days after the first ACS dose (pooled OR 0.40 ; $95 \%$ CI 0.18 to 0.89 ) and among multicentre studies (pooled OR 0.66; 95\% CI 0.46 to 0.93) (online supplemental table S5). The GRADE assessment indicated that the overall quality of evidence for the risk of IVH and PVL was very low due to the very serious inconsistency (online supplemental table S6).

\section{NEC, ROP and BPD}

We detected no difference in NEC (pooled OR 1.02; $95 \%$ CI 0.76 to $1.36 ; \mathrm{I}^{2}=67.2 \%$; $\mathrm{p}=0.006$ ), ROP (pooled OR $1.01 ; 95 \%$ CI 0.94 to $1.08 ; \mathrm{I}^{2}=38.7 \% ; \mathrm{p}=0.134$ ) or BPD (pooled OR 1.00; 95\% CI 0.81 to $1.23 ; \mathrm{I}^{2}=53.3 \%$; $\mathrm{p}=0.036$ ) between multiple newborns with and those without ACS (table 1, online supplemental figure S5S7). We did not find any significantly decreased pooled ORs between subgroups, based on ACS completeness, administration-to-delivery intervals and multicentre or single-centre studies (online supplemental table S5). The GRADE assessment indicated that the overall quality of evidence for the risk of NEC was very low due to the very serious inconsistency and that for the risk of ROP and BPD was low due to the serious inconsistency (online supplemental table S6).

\section{Sensitivity and publication bias analyses}

To assess the robustness of the current findings, sensitivity analyses were performed by omitting individual studies. The results confirmed that the pooled estimates of each outcome were reliable (available in online supplemental figure S8-S14).

The Begg's and Egger's tests showed no any evidence of publication bias on any outcomes ( $p>0.05)$, except RDS (Egger's test, $\mathrm{p}=0.015$ ) (table 1). The Egger's publication bias plots for neonatal outcomes were shown in online supplemental figure S15-S21). Then we performed sensitivity analysis using the trim and fill method to assess the possibility of publication bias. No trimming was performed, and the data were unchanged throughout the analysis, suggesting the absence of publication bias for the association between ACS and RDS.

\section{DISCUSSION}

In the current meta-analysis, based on the very low-quality evidence, we found that ACS treatment may be associated with a reduction in RDS, neonatal mortality, IVH and PVL among multiple PTB, despite the substantial heterogeneity. There was no difference in NEC, ROP or BPD between preterm multiples with and without ACS treatment. Different associations of ACS treatment and RDS, mortality and PVL were found between subgroups in terms of ACS completeness, administration-to-delivery interval as well as multicentre or single-centre study. The sensitivity analyses confirmed the robustness of the current results and no publication bias was found.

The prior evidence of the efficacy of ACS among singletons has been abundant, but evidence among multiple births has been sparse and limited to small observational studies. No evidence of randomised trials on the efficacy of ACS in multiple pregnancies is currently available. We retrieved only one RCT protocol by Hong et $a l^{39}$ despite no restriction on study design in the literature search. Among these observational studies, the quality of evidence for outcomes of interest was categorised as very low or low, mainly due to huge inconsistency across studies, according to the GRADE criteria. In this regard, it is necessary to investigate the impact of ACS on the outcomes among multiple gestations.

ACS has been confirmed to be effective to prepare fetal lung for air breathing through multiple mechanisms, including the induction of protein and enzymes, the acceleration of antioxidant production and induction of beta-receptor expression in alveolar cells as well as the acceleration of parenchymal change. ${ }^{40}$ Though ACS has been internationally recommended in clinical practice, there is no difference in the guidelines for administration of ACS between multiple gestations and singletons. ${ }^{11}{ }^{41}$ From the view of pharmacokinetics, however, shorter half-life and faster clearance of corticosteroids in multiple pregnancies might raise some doubts in the effectiveness of ACS among multiple pregnancies when using the regime as same as for singletons. ${ }^{42}$ Overall, we found benefits of ACS to reduce RDS, IVH, PVL and mortality based on the combined results, but no difference in NEC, ROP and BPD. These results were similar to previous ones obtained from singletons regarding RDS, IVH, PVL and neonatal death but not regarding NEC, ROP and BPD. ${ }^{104-45}$ It was not surprising to detect substantial heterogeneity since there was variation in the exclusion criteria as well as the definition of outcomes. In the subgroup analysis, a complete ACS course was found to be attributed to a reduction in RDS, mortality and PVL. In contrast, no benefit was found in these outcomes when 
those studies had no information on ACS completeness. Based on the assumption that these studies could include both populations with and without a complete ACS, we thought that ACS completeness could play a role in heterogeneity. Although the efficacy of incomplete ACS was shown by a previous study conducted in singletons, ${ }^{46}$ it remained challenged by gestational age at delivery. ${ }^{47}$ Herrera $e t a t^{21}$ did not show a significant reduction in the risks of death and RDS among multiple gestations exposed to incomplete ACS. The ACS administration-to-delivery interval was also regarded as a source of heterogeneity, since a significant reduction of RDS and PVL was found among preterm twins delivered within 7 days after administration but not among those without information on administration timing. To date, it has been recommended that the corticosteroids should be administered within 7 days prior to PTB, respective of plurality. ${ }^{41}{ }^{48-51} \mathrm{Kuk}$ et $a l^{20}$ compared RDS between twins without treatments and those with ACS-to-delivery intervals of $<2,2-7$ and $>7$ days. The reduction in RDS was only observed in the group with an ACS-to-delivery interval of 2-7 days but not in the other groups. A retrospective cohort of 106 twin pregnancies with suspected PTB found that newborns born beyond 7 days after the ACS course experienced increased composite respiratory complications in comparison to those born within 7 days after ACS treatment. ${ }^{52}$ We still found a reduction of other adverse outcomes even though the studies were thought to include populations with administration-to-delivery intervals of $>7$ days. A retrospective study by Rottenstreich et $a \tilde{p}^{\tilde{3}}$ demonstrated that the rate of optimal initial ACS (ACS administration-to-delivery interval $\geq 24$ hour and $\leq 7$ days) was significantly lower in twin pregnancies than in singletons $(19.7 \%$ vs $33.2 \%, \mathrm{p}=0.001)$. It was a challenge to predict PTB among multiple gestations, which hampers the healthcare providers from achieving optimal ACS administration. In this regard, a more robust model of prediction for PTB among multiple gestations is expected to provide evidence for ACS administration.

There were several limitations that should be considered in the present meta-analysis. First, the majority of included studies were retrospective in design, suggesting that some bias in retrospective nature cannot be avoided and the overall quality of evidence was low, which may impede the interpretation of the current results. Higher levels of evidence on the efficacy of ACS among preterm twins are of great need. ${ }^{39}$ Second, the high heterogeneity may weaken the validity of current results. Even though subgroup analyses were performed, the high heterogeneity remained in some subgroups, suggesting the presence of unexplained heterogeneity. Third, we were unable to perform subgroup analysis on gestational age at delivery because of the substantial variation in this information. Overall, the studies included preterm pregnancies before 35 weeks of gestational age except two, ${ }^{158}$ and the sensitivity analysis confirmed the robustness of pooled results after omitting these studies, however.

\section{CONCLUSIONS}

Our meta-analysis of observational studies suggests that a complete course of ACS treatment within 7 days of delivery is beneficial to reduce neonatal complications in terms of RDS, mortality, IVH and PVL among preterm multiple births. ACS is not associated with a reduction in NEC, ROP or BPD. ACS completeness, administration-to-delivery interval and multicentre study could play a role in the heterogeneity. More robust evidence on the efficacy of ACS treatment among multiple gestations is warranted.

Acknowledgements The authors thank the researchers of included studies for sharing their knowledge.

Contributors $\mathrm{DL}$ and $\mathrm{CL}$ contributed to the study conception and drafted the protocol. GC and DF contributed to study selection and data collection; DL performed data analysis and drafted the manuscript. $C L$ and $X G$ contributed to interpretation. ZL contributed to manuscript revision. All authors have read and approved the manuscript.

Funding The authors have not declared a specific grant for this research from any funding agency in the public, commercial or not-for-profit sectors.

Competing interests None declared.

Patient consent for publication Not required.

Provenance and peer review Not commissioned; externally peer reviewed.

Data availability statement Data are available upon reasonable request.

Supplemental material This content has been supplied by the author(s). It has not been vetted by BMJ Publishing Group Limited (BMJ) and may not have been peer-reviewed. Any opinions or recommendations discussed are solely those of the author(s) and are not endorsed by BMJ. BMJ disclaims all liability and responsibility arising from any reliance placed on the content. Where the content includes any translated material, BMJ does not warrant the accuracy and reliability of the translations (including but not limited to local regulations, clinical guidelines, terminology, drug names and drug dosages), and is not responsible for any error and/or omissions arising from translation and adaptation or otherwise.

Open access This is an open access article distributed in accordance with the Creative Commons Attribution Non Commercial (CC BY-NC 4.0) license, which permits others to distribute, remix, adapt, build upon this work non-commercially, and license their derivative works on different terms, provided the original work is properly cited, appropriate credit is given, any changes made indicated, and the use is non-commercial. See: http://creativecommons.org/licenses/by-nc/4.0/.

\section{ORCID iDs}

Caihong Luo http://orcid.org/0000-0002-1289-0325

Zhengping Liu http://orcid.org/0000-0002-4964-0258

\section{REFERENCES}

1 Ibiebele I, Humphries JB, Torvaldsen S, et al. Gestational age, morbidity and mortality among twin births in New South Wales, Australia 2003-2014: a cohort study. Aust N Z J Obstet Gynaecol 2020;60:541-7.

2 Garg P, Abdel-Latif ME, Bolisetty S, et al. Perinatal characteristics and outcome of preterm singleton, twin and triplet infants in NSW and the act, Australia (1994-2005). Arch Dis Child Fetal Neonatal Ed 2010;95:F20-4.

$3 \mathrm{Ko} \mathrm{HS}$, Wie JH, Choi SK, et al. Multiple birth rates of Korea and fetal/neonatal/infant mortality in multiple gestation. PLoS One 2018;13:e0202318.

4 Martin JA, Hamilton BE, Osterman MJK. Births: final data for 2018. National vital statistics reports : from the Centers for Disease Control and Prevention, National Center for Health Statistics, National Vital Statistics System 2019;68:1-47.

5 van Zijl MD, Koullali B, Oudijk MA, et al. Trends in preterm birth in singleton and multiple gestations in the Netherlands 2008-2015: a population-based study. Eur J Obstet Gynecol Reprod Biol 2020;247:111-5

6 Ma R, Luo Y, Wang J, et al. Ten-Year time trends in preterm birth during a sociodemographic transition period: a retrospective cohort study in Shenzhen, China. BMJ Open 2020;10:e037266. 
7 Ness A, Mayo JA, El-Sayed YY, et al. Trends in spontaneous and medically indicated preterm birth in twins versus singletons: a California cohort 2007 to 2011. Am J Perinatol 2021. doi:10.1055/s-0041-1729161. [Epub ahead of print: 02 May 2021].

8 Burger RJ, Temmink S, Wertaschnigg D, et al. Trends in preterm birth in twin pregnancies in Victoria, Australia, 2007-2017. Aust N Z J Obstet Gynaecol 2021;61:55-62.

9 Liggins GC, Howie RN. A controlled trial of antepartum glucocorticoid treatment for prevention of the respiratory distress syndrome in premature infants. Pediatrics 1972;50:515-25.

10 Roberts D, Brown J, Medley N. Antenatal corticosteroids for accelerating fetal lung maturation for women at risk of preterm birth. Cochrane Database of Systematic Reviews 2017.

11 Committee on Practice Bulletins - Obstetrics, Society for MaternalFetal Medicine. Practice Bulletin No. 169: multifetal gestations: twin, triplet, and higher-order multifetal pregnancies. Obstet Gynecol 2016;128:e131-46.

12 Health NCCFWSS. Multiple pregnancy: the management of twin and triplet pregnancies in the antenatal period | information for the public. NICE, 2011.

13 Thevathasan I, Said JM. Controversies in antenatal corticosteroid treatment. Prenat Diagn 2020;40:1138-49.

14 Blickstein I. Antenatal corticosteroids: current controversies. $J$ Perinat Med 2017;45:5-9.

15 Viteri OA, Blackwell SC, Chauhan SP, et al. 527: antenatal corticosteroids do not reduce the incidence of respiratory distress syndrome in preterm triplets. Am J Obstet Gynecol 2016;214:S284-5.

$16 \mathrm{Vaz}$ A, Malheiro MF, Severo M, et al. Effect of antenatal corticosteroids on morbidity and mortality of preterm singletons and twins. J Matern Fetal Neonatal Med 2018;31:754-60.

17 Ushida T, Kotani T, Sadachi R, et al. Antenatal corticosteroids and outcomes in preterm twins. Obstet Gynecol 2020;135:1387-97.

18 Riskin-Mashiah S, Reichman B, Bader D, et al. Population-Based study on antenatal corticosteroid treatment in preterm small for gestational age and non-small for gestational age twin infants. $J$ Matern Fetal Neonatal Med 2018;31:553-9.

19 Melamed N, Shah J, Yoon EW. The role of antenatal corticosteroids in twin pregnancies complicated by preterm birth. Am J Obstet Gynecol 2016;215

20 Kuk J-Y, An J-J, Cha H-H, et al. Optimal time interval between a single course of antenatal corticosteroids and delivery for reduction of respiratory distress syndrome in preterm twins. Am J Obstet Gynecol 2013;209:256.e1-256.e7.

21 Herrera TI, Vaz Ferreira MC, Toso A, et al. Neonatal outcomes of antenatal corticosteroids in preterm multiple pregnancies compared to singletons. Early Hum Dev 2019;130:44-50.

22 Gagliardi L, Lucchini R, Bellù R, et al. Antenatal corticosteroid prophylaxis in singleton and multiple pregnancies. Paediatr Perinat Epidemiol 2017:31:394-401.

23 Choi S-J, Song SE, Seo ES, et al. The effect of single or multiple courses of antenatal corticosteroid therapy on neonatal respiratory distress syndrome in singleton versus twin pregnancies. Aust N $Z J$ Obstet Gynaecol 2009;49:173-9.

24 Boghossian NS, McDonald SA, Bell EF, et al. Association of antenatal corticosteroids with mortality, morbidity, and neurodevelopmental outcomes in extremely preterm multiple gestation infants. JAMA Pediatr 2016;170:593-601.

25 Beksac MS, Korkmaz A, Kasapoglu T. Antenatal corticosteroids for women at risk of preterm delivery: the "Emperor's New Clothes" tale in medical practice. Journal of Maternal-Fetal and Neonatal Medicine 2020.

26 Moher D, Liberati A, Tetzlaff J, et al. Preferred reporting items for systematic reviews and meta-analyses: the PRISMA statement. PLoS Med 2009;6:e1000097.

27 Stroup DF, Berlin JA, Morton SC, et al. Meta-Analysis of observational studies in epidemiology: a proposal for reporting. meta-analysis of observational studies in epidemiology (moose) group. JAMA 2000;283:2008-12.

28 Guyatt G, Oxman AD, Akl EA, et al. Grade guidelines: 1. IntroductionGRADE evidence profiles and summary of findings tables. J Clin Epidemiol 2011;64:383-94.

29 Papile LA, Burstein J, Burstein R, et al. Incidence and evolution of subependymal and intraventricular hemorrhage: a study of infants with birth weights less than 1,500 GM. J Pediatr 1978;92:529-34.

30 Bell MJ, Ternberg JL, Feigin RD, et al. Neonatal necrotizing enterocolitis. therapeutic decisions based upon clinical staging. Ann Surg 1978;187:1-7.
31 International Committee for the Classification of Retinopathy of Prematurity. The International classification of retinopathy of prematurity revisited. Arch Ophthalmol 2005;123:991-9.

$32 \mathrm{Xu} \mathrm{T}$, Zhang Y-H. Association of psoriasis with stroke and myocardial infarction: meta-analysis of cohort studies. $\mathrm{Br} J$ Dermatol 2012;167:1345-50.

33 Borenstein M, Hedges LV, Higgins JPT, et al. A basic introduction to fixed-effect and random-effects models for meta-analysis. Res Synth Methods 2010;1:97-111

34 Turrentine MA, Wilson PD, Wilkins IA. A retrospective analysis of the effect of antenatal steroid administration on the incidence of respiratory distress syndrome in preterm twin pregnancies. Am J Perinatol 1996;13:351-4

35 Spinillo A, Capuzzo E, Ometto A, et al. Value of antenatal corticosteroid therapy in preterm birth. Early Hum Dev 1995;42:37-47.

36 Palas D, Ehlinger V, Alberge C, et al. Efficacy of antenatal corticosteroids in preterm twins: the EPIPAGE-2 cohort study. BJOG 2018;125:1164-70.

37 Kong X, Xu F, Wang Z, et al. Antenatal corticosteroids administration on mortality and morbidity in premature twins born at 25 34 gestational weeks: a retrospective multicenter study. Eur J Obstet Gynecol Reprod Biol 2020;253:259-65.

38 Ben-David A, Zlatkin R, Bookstein-Peretz S, et al. Does antenata steroids treatment in twin pregnancies prior to late preterm birth reduce neonatal morbidity? Evidence from a retrospective cohort study. Arch Gynecol Obstet 2020;302:1121-6.

39 Hong S, Lee SM, Kwak DW, et al. Effects of antenatal corticosteroids in twin neonates with late preterm birth (ACTWIN [Antenatal Corticosteroids in TWIN late preterm neonates] trial): study protocol for a randomized controlled trial. BMC Pregnancy Childbirth 2019;19:19.

40 Wapner RJ. Antenatal corticosteroids for periviable birth. Semin Perinatol 2013;37:410-3.

41 Committee on Obstetric Practice. Committee opinion no. 713 antenatal corticosteroid therapy for fetal maturation. Obstet Gynecol 2017:130:e102-9.

42 Ballabh P, Lo ES, Kumari J, et al. Pharmacokinetics of betamethasone in twin and singleton pregnancy. Clin Pharmacol Ther 2002;71:39-45.

43 McGoldrick E, Stewart F, Parker R, et al. Antenatal corticosteroids for accelerating fetal lung maturation for women at risk of preterm birth. Cochrane Database Syst Rev 2020;12:Cd004454.

44 Deshmukh M, Patole S. Antenatal corticosteroids in impending preterm deliveries before 25 weeks' gestation. Arch Dis Child Fetal Neonatal Ed 2018;103:F173-6.

45 Travers $\mathrm{CP}$, Clark RH, Spitzer AR, et al. Exposure to any antenatal corticosteroids and outcomes in preterm infants by gestational age: prospective cohort study. BMJ 2017;356:j1039.

46 Elimian A, Figueroa R, Spitzer AR, et al. Antenatal corticosteroids: are incomplete courses beneficial? Obstet Gynecol 2003;102:352-5.

47 Costa S, Zecca E, De Luca D, et al. Efficacy of a single dose of antenatal corticosteroids on morbidity and mortality of preterm infants. Eur J Obstet Gynecol Reprod Biol 2007;131:154-7.

48 National Institute for Health and Care Excellence. Nice guideline 25: preterm labour and birth, 2015. Available: https://www.nice. org.uk/guidance/ng25/resources/preterm-labour-and-birth-pdf1837333576645

49 Skoll A, Boutin A, Bujold E, et al. No. 364-Antenatal corticosteroid therapy for improving neonatal outcomes. J Obstet Gynaecol Can 2018;40:1219-39.

50 WHO Guidelines Approved by the Guidelines Review Committee. Who recommendations on interventions to improve preterm birth outcomes. 2015. Geneva: World Health Organization Copyright (C) World Health Organization, 2015.

51 FIGO Working Group on Good Clinical Practice in Maternal-Fetal Medicine. Good clinical practice advice: antenatal corticosteroids for fetal lung maturation. Int J Gynaecol Obstet 2019;144:352-5.

52 Kosinska-Kaczynska K, Szymusik I, Urban P, et al. Relation between time interval from antenatal corticosteroids administration to delivery and neonatal outcome in twins. J Obstet Gynaecol Res 2016;42:625-31.

53 Rottenstreich A, Levin G, Kleinstern G, et al. Patterns of use and optimal timing of antenatal corticosteroids in twin compared with singleton pregnancies. Acta Obstet Gynecol Scand 2018;97:1508-14. 